\title{
A REMARK ON KRONECKER'S THEOREM ON FORMS
}

\section{HARLEY FLANDERS}

The Kronecker theorem on forms over an integral domain is a consequence of integral closure. We refer the reader to [1 ] ${ }^{1}$ for a proof and other references. We have failed to find in the literature a statement of the converse of this result and consequently shall here prove that integral closure is a consequence of a relatively weak form [2] of Kronecker's theorem.

Let $\mathcal{O}$ be an integral domain which has the following property: Whenever each coefficient of the product of a linear polynomial $f(X)=a_{0} X+a_{1}$ of $\mathcal{O}[X]$ by an arbitrary polynomial $g(X)=b_{0} X^{n}+\cdots+b_{n}$ of $\mathcal{O}[X]$ is divisible by an element $c$ of $\mathcal{O}$, then $a_{1} b_{0}$ is divisible by $c$. Then $\mathcal{O}$ is integrally closed in its quotient field $k$.

To prove this, we let $\alpha=u / v \in k, u, v \in \mathcal{O}$, and assume $\alpha$ is integral over $\mathcal{O}$. Thus $h(\alpha)=0$ where

$$
h(X)=X^{m}+c_{1} X^{m-1}+\cdots+c_{m}
$$

with all $c_{j} \in \mathcal{O}$. Clearly

$$
h(X)=(X-\alpha)\left(X^{m-1}+\beta_{1} X^{m-2}+\cdots+\beta_{m-1}\right)
$$

with $\beta_{j} \in k$. We select $w \in \mathcal{O}$ such that $w \beta_{j} \in \mathcal{O}$ for all $j$ and have

$$
v w h(X)=(v X-u)\left(w X^{m-1}+w \beta_{1} X^{m-2}+\cdots+w \beta_{m-1}\right) .
$$

Since $v w$ divides each coefficient of the left-hand side of this equation, it follows from the hypothesis that $v w$ divides $u w$, and hence $v$ divides $u$. This implies that $\alpha$ is in $\mathcal{O}$, which completes the proof.

\section{REFERENCES}

1. W. Krull, Idealtheorie, Ergebnisse der Mathematik vol. 4, no. 3, 1935, especially $\$ 45$.

2. H. Weyl, Algebraic theory of numbers, Princeton, 1940, especially Lemma II 7 , A, p. 55 .

California Institute of Technology

Received by the editors August 6, 1951.

1 Numbers in brackets refer to the references at the end of the paper. 